\title{
Superconformal mechanics and nonlinear supersymmetry
}

\author{
Carlos Leiva $^{a *}$ and Mikhail S. Plyushchay ${ }^{a, b \dagger}$ \\ ${ }^{a}$ Departamento de Física, Universidad de Santiago de Chile, Casilla 30\%, Santiago 2, Chile \\ ${ }^{b}$ Institute for High Energy Physics, Protvino, Russia
}

\begin{abstract}
We show that a simple change of the classical boson-fermion coupling constant, $2 \alpha \rightarrow 2 \alpha n, n \in \mathbb{N}$, in the superconformal mechanics model gives rise to a radical change of a symmetry: the modified classical and quantum systems are characterized by the nonlinear superconformal symmetry. It is generated by the four bosonic integrals which form the $s o(1,2) \times u(1)$ subalgebra, and by the $2(n+1)$ fermionic integrals constituting the two spin- $\frac{n}{2}$ so $(1,2)$-representations and anticommuting for the order $n$ polynomials of the even generators. We find that the modified quantum system with an integer value of the parameter $\alpha$ is described simultaneously by the two nonlinear superconformal symmetries of the orders relatively shifted in odd number. For the original quantum model with $|\alpha|=p, p \in \mathbb{N}$, this means the presence of the order $2 p$ nonlinear superconformal symmetry in addition to the $\operatorname{sep}(2 \mid 2)$ supersymmetry.
\end{abstract}

\footnotetext{
${ }^{*}$ E-mail: caleiva@lauca.usach.cl

${ }^{\dagger}$ E-mail: mplyushc@lauca.usach.cl
} 


\section{Introduction}

The conformal mechanics model of De Alfaro, Fubini and Furlan [1] was introduced and studied as a $0+1$-dimensional conformal field theory. Its supersymmetric analog was constructed by Akulov and Pashnev [2], and by Fubini and Rabinovici [3], while the geometric nature of the original and superextended versions of the model was established by Ivanov, Krivonos and Leviant [4]. A recent revival of interest in (super)conformal mechanics [5]-[22] has been triggered, in particular, by an observation made in the context of AdS/CFT correspondence conjecture [23] that the dynamics of a superparticle near the AdS horizon of an extreme Reissner-Nordström black hole is described by a superconformal mechanics [5].

There is, on the other hand, an ongoing interest in nonlinear generalization of supersymmetric quantum mechanics [24]-[36]. It is caused mainly by the exact and quasi-exact solvability aspects of the construction [29, 30,32], and by a recent observation of a reach algebraic structure underlying nonlinear supersymmetry [31]. Such a generalization, however, has not touched yet the superconformal symmetry.

The aim of the present paper is to investigate the superconformal mechanics in the light of nonlinear supersymmetry. A priori it is not obvious whether the corresponding generalization of the superconformal mechanics exist at least at the quantum level. The reason is that unlike the standard supersymmetric mechanics possessing a linear superalgebraic structure [37], its nonlinear version is characterized by the supercharges anticommuting for the polynomials of the Hamiltonian and of additional even integrals if the latter exist [31]. Due to a nonlinearity of the supercharges in the momenta, generally a quantum anomaly prevents a preservation of nonlinear supersymmetry at the quantum level $[28,29]$. We find that not only a nontrivial and unexpected structure appears in the nonlinear generalization of superconformal mechanics already at the classical level: the set of odd integrals is extended in comparison with the original model [2,3] (cf. with nonlinear supersymmetry of refs. [29][33],[36]), but that the modified system is quantizable in an anomaly free way. Surprisingly, we find also that at the special values of the boson-fermion coupling constant, in addition to the linear $\operatorname{osp}(2 \mid 2)$ Lie superalgebraic structure, the original quantum model $[2,3]$ itself possesses the nonlinear superconformal symmetry has not been known before.

The paper is organized as follows. In Section 2 we review shortly the $\operatorname{osp}(2 \mid 2)$ Lie superalgebraic structure of the classical version of the model $[2,3]$, and construct the modified classical system having the nonlinear superconformal symmetry. We fix a general form of the corresponding nonlinear Poisson bracket structure and then, in Section 3, investigate the quantum version of the modified system and its nonlinear symmetry. In Section 4 we find that there exist some special values of the boson-fermion coupling constant, for which the quantum system is characterized simultaneously by the two nonlinear superconformal symmetries of different orders. In Section 5 we summarize the obtained results and discuss some open problems to be interesting for further investigation.

\section{Nonlinear classical superconformal mechanics}

In this section by an appropriate change of the boson-fermion coupling constant, we construct a classical modification of the superconformal mechanics model $[2,3]$ which is characterized 
by the odd integrals of motion to be polynomial in the momentum. By explicit calculation we fix the general Poisson structure of the nonlinear superconformal symmetry of the modified system.

\subsection{Linear superconformal mechanics}

To start, let us consider a free nonrelativistic particle with spin described by the Lagrangian

$$
L_{0}=\frac{1}{2} \dot{x}^{2}-\frac{i}{2} \dot{\psi}_{a} \psi_{a}
$$

Its Hamiltonian has the same form as that for the spinless particle, $H=\frac{1}{2} p^{2}$. In accordance with the equations of motion, $\frac{d A}{d t}=\frac{\partial A}{\partial t}+\{A, H\}$, generated via the Poisson-Dirac brackets $\{x, p\}=1,\left\{\psi_{a}, \psi_{b}\right\}=-i \delta_{a b}, a, b=1,2$, the quantities

$$
p, \quad X=x-t p, \quad \psi_{a}, \quad a=1,2,
$$

form the set of integrals of motion linear in phase space variables. The integrals (2.1) generate the space translations $(p)$, the Galilei boosts $(X)$, and the translations of odd variables $\left(\psi_{a}\right)$. The quadratic combinations of (2.1),

$$
\begin{gathered}
H=\frac{1}{2} p^{2}, \quad D=\frac{1}{2} X p=\frac{1}{2} x p-t H, \quad K=\frac{1}{2} X^{2}=\frac{1}{2} x^{2}-2 t D-t^{2} H, \\
\Sigma=\psi^{+} \psi^{-}=-i \psi_{1} \psi_{2}, \\
Q_{a}=p \psi_{a}, \quad S_{a}=X \psi_{a}=x \psi_{a}-t Q_{a},
\end{gathered}
$$

are also integrals of motion. The even quadratic integrals generate the translations in time $(H)$, the scale (dilatation) transformations $(D)$, the special conformal transformations $(K)$, and the rotations of Grassmann two-dimensional vector $\psi_{a}$, or, equivalently, the $\mathrm{U}(1)$ phase transformations of the complex Grassmann variables $\psi^{ \pm}(\Sigma), \psi^{ \pm}=\frac{1}{\sqrt{2}}\left(\psi_{1} \pm\right.$ $\left.i \psi_{2}\right), \quad\left\{\psi^{+}, \psi^{-}\right\}=-i$. The odd integrals $Q_{a}$ and $S_{a}$ generate the supertransformations being the square roots from the time translations, and from the special conformal transformations, respectively. The quadratic integrals form the classical supeconformal algebra $o s p(2 \mid 2) \cong s u(1,1 \mid 1)$ (we write down only the nontrivial bracket relations):

$$
\begin{gathered}
\{H, K\}=-2 D, \quad\{D, H\}=H, \quad\{D, K\}=-K, \\
\left\{Q_{a}, Q_{b}\right\}=-2 i \delta_{a b} H, \quad\left\{S_{a}, S_{b}\right\}=-2 i \delta_{a b} K, \\
\left\{S_{a}, Q_{b}\right\}=-2 i \delta_{a b} D+i \epsilon_{a b} \Sigma, \\
\left\{H, S_{a}\right\}=-Q_{a}, \quad\left\{K, Q_{a}\right\}=S_{a}, \\
\left\{D, Q_{a}\right\}=\frac{1}{2} Q_{a}, \quad\left\{D, S_{a}\right\}=-\frac{1}{2} S_{a}, \\
\left\{\Sigma, Q_{a}\right\}=\epsilon_{a b} Q_{b}, \quad\left\{\Sigma, S_{a}\right\}=\epsilon_{a b} S_{b} .
\end{gathered}
$$

One could construct higher order even and odd composite in $p, X$ and $\psi^{ \pm}$integrals of motion. In such a way, e.g., the super-Virasoro algebra can be realized as a symmetry on the phase space of the free spin particle, see refs. $[10,11]$. 
The case of the superconformal mechanics given by the Hamiltonian

$$
H=\frac{1}{2}\left(p^{2}+\frac{1}{x^{2}} \alpha\left(\alpha+2 i \psi_{1} \psi_{2}\right)\right)
$$

is less trivial: for such a system none of the linear variables (2.1) is the integral of motion, but there exist the analogs of the quadratic integrals (2.2)-(2.4) which form the same superconformal algebra $(2.5)^{1}$. The corresponding generators of the subalgebra $s o(1,2) \times u(1)$ are the Hamiltonian (2.6), and

$$
\begin{gathered}
D=\frac{1}{2} x p-t H, \quad K=\frac{1}{2} x^{2}-2 t D-t^{2} H, \\
\Sigma=\alpha-i \psi_{1} \psi_{2} .
\end{gathered}
$$

The odd generators of the $\operatorname{osp}(2 \mid 2)$ take here the form

$$
\begin{gathered}
Q_{a}=p \psi_{a}+\frac{\alpha}{x} \epsilon_{a b} \psi_{b}, \\
S_{a}=(x-t p) \psi_{a}-\frac{\alpha}{x} t \epsilon_{a b} \psi_{b} .
\end{gathered}
$$

All these integrals are polynomials in $p$ of order not higher than 2 , and so, their quantum analogs are the local differential operators.

\subsection{Nonlinear generalization}

Now, following ref. [28] we shall show that a simple change of the boson-fermion coupling constant, $2 \alpha \rightarrow 2 \alpha n, n \in \mathbb{N}$, i.e. the change of (2.6) for the Hamiltonian

$$
H_{n}=\frac{1}{2}\left(p^{2}+\frac{1}{x^{2}} \alpha\left(\alpha+2 i n \psi_{1} \psi_{2}\right)\right)
$$

gives rise to a radical change of the symmetry: instead of the linear symmetry associated with the Lie superalgebra $\operatorname{osp}(2 \mid 2)$, the system (2.10) is characterized by the nonlinear superconformal symmetry containing the same even subalgebra $s o(1,2) \times u(1)$, but whose odd part includes $2(n+1)$ generators commuting (in the sense of classical Poisson brackets, or, of quantum anticommutators) for polynomials of even generators.

To reveal and analyze the superalgebraic structure of the system (2.10), we define the even oscillator-like variables

$$
z=\frac{\alpha}{x}+i p, \quad \bar{z}=\frac{\alpha}{x}-i p, \quad\{z, \bar{z}\}=2 i \frac{\alpha}{x^{2}}
$$

in terms of which the Hamiltonian (2.10) takes the form $H_{n}=\frac{1}{2}\left(z \bar{z}+i n\{z, \bar{z}\} \psi^{+} \psi^{-}\right)$. In addition to the Hamiltonian and nilpotent charge

$$
\Sigma=\psi^{+} \psi^{-}
$$

\footnotetext{
${ }^{1}$ The super-Virasoro algebra may also be realized as a symmetry on the phase space of the system (2.6), see refs. [10, 18].
} 
the system (2.10) is characterized by the even integrals $D_{n}$ and $K_{n}$ of the form (2.7) (with the Hamiltonian (2.10)). But, instead of the odd quantities (2.8), here we have the integrals

$$
S_{n, 0}^{+}=z^{n} \psi^{+}, \quad S_{n, 0}^{-}=\bar{z}^{n} \psi^{-},
$$

whereas the analogs of the explicitly depending on $t$ integrals of motion (2.9) are given by

$$
S_{n, 1}^{+}=(x+i t z) z^{n-1} \psi^{+}, \quad S_{n, 1}^{-}=(x-i t \bar{z}) \bar{z}^{n-1} \psi^{-} .
$$

More exactly, at $n=1$ the integrals (2.12), (2.13) take the form of the linear combinations of the integrals (2.8), (2.9): $S_{1,0}^{+}=\frac{i}{\sqrt{2}}\left(Q_{1}+i Q_{2}\right), S_{1,1}^{+}=\frac{1}{\sqrt{2}}\left(S_{1}+i S_{2}\right)$. The odd integrals (2.13) are different from the integrals (2.12) in the factor

$$
\frac{x}{z}+i t=\frac{\alpha-2 i D_{n}}{2 H_{n}}
$$

and in its complex conjugate, which is the even (explicitly depending on time) integral of motion. Then, one can extend the odd integrals (2.12), (2.13) by the set of odd integrals of the form

$$
S_{n, l}^{+}=(x+i t z)^{l} z^{n-l} \psi^{+}=\left(\frac{\alpha-2 i D_{n}}{2 H_{n}}\right)^{l} S_{n, 0}^{+}, \quad S_{n, l}^{-}=\left(S_{n, l}^{+}\right)^{*},
$$

where $l=2, \ldots, n$. The first equality from (2.14) means that all the integrals $S_{n, l}^{ \pm}, l=$ $0,1, \ldots, n$, are polynomials of the order $n$ in the momentum $p$. Therefore, forgetting the anomaly problem [28, 29], their quantum analogs have to be the local differential operators of the order $n$. The second representation of $S_{n, l}^{+}$from (2.14) will be helpful in finding the explicit form of the superalgebra formed by all the set of the even, $H_{n}, D_{n}, K_{n}$, $\Sigma$, and the odd, $S_{n, l}^{ \pm}, l=0, \ldots, n$, integrals, from which it will be clear why the set of the integrals (2.12), (2.13) has to be extended by the integrals $(2.14)$ with $l=2, \ldots, n$.

First, we find that the bosonic part of the superalgebra (2.5) does not change,

$$
\left\{H_{n}, K_{n}\right\}=-2 D_{n}, \quad\left\{D_{n}, H_{n}\right\}=H_{n}, \quad\left\{D_{n}, K_{n}\right\}=-K_{n} .
$$

Then, taking into account the brackets $\left\{S_{n, 0}^{+}, S_{n, 0}^{-}\right\}=-i(2 H)^{n}$, and the relations

$$
\begin{gathered}
4\left(D_{n}^{2}-K_{n} H_{n}\right)-2 n \alpha \Sigma=-\alpha^{2}, \\
S_{n, 0}^{+} S_{n, 0}^{-}=\left(2 H_{n}\right)^{n} \Sigma,
\end{gathered}
$$

one can find all other nontrivial brackets of the integrals:

$$
\begin{gathered}
\left\{D_{n}, S_{n, l}^{ \pm}\right\}=\left(\frac{n}{2}-l\right) S_{n, l}^{ \pm}, \quad\left\{\Sigma, S_{n, l}^{ \pm}\right\}=\mp i S_{n, l}^{ \pm}, \\
\left\{H_{n}, S_{n, l}^{ \pm}\right\}= \pm i l S_{n, l-1}^{ \pm}, \quad\left\{K_{n}, S_{n, l}^{ \pm}\right\}= \pm i(n-l) S_{n, l+1}^{ \pm}, \\
\left\{S_{n, m}^{+}, S_{n, l}^{-}\right\}=\quad \begin{array}{l}
-i\left(2 H_{n}\right)^{n-m}\left(2 K_{n}\right)^{l}(\alpha-2 i D)^{m-l}-i \Sigma\left(2 H_{n}\right)^{n-m-1}\left(2 K_{n}\right)^{l-1} \times \\
\left(\alpha-2 i D_{n}\right)^{m-l}\left(n(m-l)\left(\alpha-2 i D_{n}\right)+4 \alpha l(n-m)\right), \quad m \geq l .
\end{array}
\end{gathered}
$$


The brackets $\left\{S_{n, m}^{+}, S_{n, l}^{-}\right\}$for $m<l$ may be obtained from (2.20) by complex conjugation with application of the relation $\left\{B^{*}, A^{*}\right\}=-(\{A, B\})^{*}$ valid for arbitrary phase space functions $A, B$ of fixed Grassmann parity.

The bracket relations mean that the integrals of motion of the system (2.10) form the $\mathbb{Z}_{2}$-graded nonlinear Poisson algebra: the right hand side of Eq. (2.20) is polynomial in the generators of the $s o(1,2) \times u(1)$ subalgebra.

At first sight it seems that at $l=0$ and at $m=n$ the nilpotent term (proportional to $\Sigma$ ) on the right hand side of Eq. (2.20) contains a nonpolynomial factor. However, the direct calculation with using the relation (2.16) shows that in these two cases the brackets $(2.20)$ may be reduced to the explicitly polynomial form,

$$
\begin{gathered}
\left\{S_{n, m}^{+}, S_{n, 0}^{-}\right\}=-i\left(2 H_{n}\right)^{n-m}\left(\alpha-2 i D_{n}\right)^{m-1}\left(\left(\alpha-2 i D_{n}\right)+n m \Sigma\right) \\
\left\{S_{n, n}^{+}, S_{n, l}^{-}\right\}=-i\left(2 K_{n}\right)^{l}\left(\alpha-2 i D_{n}\right)^{n-l-1}\left(\left(\alpha-2 i D_{n}\right)+n(n-l) \Sigma\right) .
\end{gathered}
$$

According to the relations (2.19), the Hamiltonian acts on the set $S_{n, l}^{ \pm}$as a decreasing in index $l$ generator, "annihilating" the lowest in $l$ odd integrals $S_{n, 0}^{ \pm}$which do not depend, unlike the $S_{n, l}^{ \pm}, 1 \leq l \leq n$, explicitly on time. On the other hand, $K_{n}$ acts as an increasing in $l$ generator annihilating the highest in $l$ odd generators $S_{n, n}^{ \pm}$. Having also in mind the first relation from (2.18), we conclude that the sets of odd generators $S_{n, l}^{+}$and $S_{n, l}^{-}$are transformed with respect to the action of the $s o(1,2)$ generators as the two $(n+1)$-dimensional multiplets (spin- $\frac{n}{2}$ "states"). Therefore, the subsequent action (via the Poisson brackets) of the conformal boost generator $K_{n}$ on the not depending explicitly on time integrals $S_{n, 0}^{ \pm}$ generate all the finite set of the odd integrals $S_{n, l}^{ \pm}$terminating the series on $l=n$.

In accordance with relations (2.16), (2.17), the classical nonlinear superconformal algebra (2.15), (2.18), (2.19), (2.20) has the Casimir elements $D_{n}^{2}-K_{n} H_{n}-\frac{1}{2} \alpha n \Sigma$ and $S_{n, 0}^{+} S_{n, 0}^{-}-\left(2 H_{n}\right)^{n} \Sigma$ taking here the values $-\frac{1}{4} \alpha^{2}$ and 0 , respectively. Analogously, the even combinations $S_{n, l}^{+} S_{n, l}^{-}-\left(2 H_{n}\right)^{n-l}(2 K)_{n}^{l} \Sigma, l=1, \ldots, n$, are also classical central elements of the algebra taking a zero value.

\section{Quantum case}

As we already mentioned, the specific feature of nonlinear supersymmetry, unlike the linear $n=1$ case, consists in appearance of the quantum anomaly problem that destroys the conservation of the odd generators at the quantum level [28, 29]. However, there exist some classes of superpotentials for which the problem of the quantum anomaly may be resolved [29]-[32]. Below we show that the system (2.10) belongs to such a class.

\subsection{Quantum integrals of motion}

Let us choose the quantum analog of the Hamiltonian (2.10) in the form

$$
\begin{gathered}
H_{n}=\frac{1}{2}\left(-\frac{d^{2}}{d x^{2}}+\frac{1}{x^{2}}\left(a_{n}+b_{n} \sigma_{3}\right)\right), \\
a_{n}=\alpha_{n}^{2}+\frac{1}{4}\left(n^{2}-1\right), \quad b_{n}=-n \alpha_{n}, \quad \alpha_{n}=\alpha-\frac{1}{2}(n-1),
\end{gathered}
$$


where $\Delta_{1}=a_{n}-\alpha^{2}$ and $\Delta_{2}=b_{n}+n \alpha$ are the quantum corrections [29] (here we put $\hbar=1$ ) which, as will be shown, guarantee the conservation of the nonlinear superconformal symmetry.

First we note that the quantum analogs of the generators (2.7), (2.11) of the classical bosonic symmetry $s o(1,2) \times u(1)$,

$$
D_{n}=\frac{1}{4}[x, p]_{+}-t H_{n}, \quad K_{n}=\frac{1}{2} x^{2}-2 t D_{n}-t^{2} H_{n}, \quad \Sigma=\frac{1}{2}\left[\psi^{+}, \psi^{-}\right]=\frac{1}{2} \sigma_{3},
$$

are the integrals of motion of the quantum system (3.1). Any of them satisfies the equation of the form

$$
i \frac{\partial I_{n}}{\partial t}-\left[H_{n}, I_{n}\right]=0
$$

The quantum system (3.1) admits also the set of odd integrals being the quantum analogs of the classical integrals $S_{n, l}^{ \pm}, l=0,1, \ldots, n$. These are given by the relations

$$
\begin{gathered}
S_{n, 0}^{+}=\mathcal{P}_{\alpha, n} \psi^{+} \\
S_{n, l}^{+}=\left(x+i t \mathcal{D}_{\alpha-n+1}\right)\left(x+i t \mathcal{D}_{\alpha-n+2}\right) \ldots\left(x+i t \mathcal{D}_{\alpha-n+l+1}\right) \mathcal{P}_{\alpha, n-l} \psi^{+}, \quad l=1, \ldots, n,
\end{gathered}
$$

where

$$
\mathcal{P}_{\alpha, n}=\mathcal{D}_{\alpha-n+1} \mathcal{D}_{\alpha-n+2} \ldots \mathcal{D}_{\alpha-1} \mathcal{D}_{\alpha}, \quad \mathcal{D}_{\gamma}=\frac{d}{d x}+\frac{\gamma}{x}, \quad \psi^{ \pm}=\frac{1}{2}\left(\sigma_{1} \pm i \sigma_{2}\right)
$$

To prove the conservation of the odd operators (3.5), (3.6), we use the relation

$$
\mathcal{D}_{\alpha-k}\left(x+i t \mathcal{D}_{\alpha-k+1}\right)=\left(x+i t \mathcal{D}_{\alpha-k}\right) \mathcal{D}_{\alpha-k+1}
$$

to get the identities

$$
\frac{\partial S_{n, l}^{+}}{\partial t}=i l S_{n, l-1}^{+}
$$

and

$$
S_{n, l}^{+}=i t S_{n, l-1}^{+}+x S_{n-1, l-1}^{+},
$$

which are valid for $l=1, \ldots, n$. Next, we note that $\left[H_{1}, S_{1,0}^{+}\right]=0$ and suppose that $\left[H_{n-1}, S_{n-1,0}^{+}\right]=0$ is valid for $n \geq 1$. Then, using the relation

$$
H_{n}=H_{n-1}+(n-\alpha-1) \frac{1}{x^{2}} \Pi_{+},
$$

where $\Pi_{+}=\frac{1}{2}\left(1+\sigma_{3}\right), \Pi_{+} \psi^{+}=\psi^{+}, \psi^{+} \Pi_{+}=0$, we find that $\left[H_{n}, S_{n, 0}^{+}\right]=0$. Therefore, by induction, we conclude that $S_{n, 0}^{+}$commutes with corresponding $H_{n}$ for any $n \in \mathbb{N}$. A simple calculation shows that $S_{n, 1}^{+}$satisfies the equation of the form (3.4), and so, is the quantum integral of motion which, like $D_{n}$ and $K_{n}$, contains an explicit dependence on time. Then, assuming that $S_{n, l-1}^{+}$satisfies equation of the form (3.4), and using the relations (3.9) and (3.10), we find that $S_{n, l}^{+}$also satisfies the same equation, and by induction conclude that $S_{n, l}^{+}$, $l=1,2, \ldots, n$, are the integrals of motion. 


\subsection{Quantum nonlinear superconformal algebra}

The quantum integrals of motion satisfy the nonlinear superconformal algebra similar to the classical one given by Eqs. (2.15), (2.18), (2.19), (2.20). Before the discussion of its structure, we note that it is sufficient to find the form of any (anti)-commutation relation for $t=0$ while its validity for arbitrary $t$ is a consequence of the unitary evolution, $I_{n}(t)=U_{n}(t) I_{n}(0) U_{n}^{-1}(t)$, $U_{n}(t)=\exp \left(-i t H_{n}\right)$, of any integral of motion $I_{n}$ obeying the equation (3.4).

The bosonic operators $(3.3)$ of the $s o(2,1) \times u(1)$ subalgebra satisfy the relations (only a nontrivial part)

$$
\left[H_{n}, K_{n}\right]=-2 i D_{n}, \quad\left[D_{n}, H_{n}\right]=i H_{n}, \quad\left[D_{n}, K_{n}\right]=-i K_{n} .
$$

Using the explicit form of the even integrals, we find that the quantum analog of the Casimir element (2.16) takes here the following value:

$$
2\left(H_{n} K_{n}+K_{n} H_{n}\right)-4 D_{n}^{2}+2 n \alpha_{n} \Sigma=\alpha_{n}^{2}+\frac{1}{4} n^{2}-1,
$$

where $\alpha_{n}$ is given by Eq. (3.2).

The nontrivial commutators between even and odd integrals are

$$
\begin{gathered}
{\left[\Sigma, S_{n, l}^{ \pm}\right]= \pm S_{n, l}^{ \pm}, \quad\left[D_{n}, S_{n, l}^{ \pm}\right]=i\left(\frac{n}{2}-l\right) S_{n, l}^{ \pm},} \\
{\left[H_{n}, S_{n, l}^{ \pm}\right]=\mp l S_{n, l-1}^{ \pm}, \quad\left[K_{n}, S_{n, l}^{ \pm}\right]=\mp(n-l) S_{n, l+1}^{ \pm} .}
\end{gathered}
$$

The commutation relations of $H_{n}$ with the odd generators follow immediately from the equations (3.4) and (3.8). The commutation relations (3.14) between $K_{n}$ and $S_{n, l}^{ \pm}$for $l=n$ and $l=n-1$ can easily be proved by a direct calculation. Then, assuming that (3.14) is valid for $l=k+1$, and using the relation

$$
S_{n, l}^{+}=\frac{1}{x} \mathcal{D}_{\alpha-n} S_{n, l+1}^{+}
$$

and its Hermitian conjugate, we prove the validity of the formula for $l=k$, that by induction finishes the proof of the second commutation relations from (3.14) for arbitrary $l, l=0, \ldots, n$.

From the dimensionality of the integrals (given by the commutator with $D_{n}$ ) it follows that the anticommutators between odd integrals coincide up to the ordering and quantum corrections to the parameter $\alpha$ (see Eq. (3.12)) with the classical relations (2.20). For the simplest nonlinear case $n=2$ these are

$$
\begin{gathered}
{\left[S_{2,0}^{+}, S_{2,0}^{-}\right]_{+}=\left(2 H_{2}\right)^{2}, \quad\left[S_{2,2}^{+}, S_{2,2}^{-}\right]_{+}=\left(2 K_{2}\right)^{2}, \quad\left[S_{2,1}^{+}, S_{2,1}^{-}\right]_{+}=\left(2 D_{2}\right)^{2}+\alpha_{2}^{2},} \\
{\left[S_{2,2}^{+}, S_{2,0}^{-}\right]_{+}=\left(2 i D_{2}-\alpha_{2}\right)\left(2 i D_{2}-\alpha_{2}-4 \Sigma\right), \quad\left[S_{2,2}^{-}, S_{2,0}^{+}\right]_{+}=\left(\left[S_{2,2}^{+}, S_{2,0}^{-}\right]_{+}\right)^{\dagger},} \\
{\left[S_{2,1}^{+}, S_{2,0}^{-}\right]_{+}=-2 i\left(D_{2} H_{2}+H_{2} D_{2}\right)+2 H_{2}\left(\alpha_{2}+2 \Sigma\right), \quad\left[S_{2,1}^{-}, S_{2,0}^{+}\right]_{+}=\left(\left[S_{2,1}^{+}, S_{2,0}^{-}\right]_{+}\right)^{\dagger},} \\
{\left[S_{2,2}^{+}, S_{2,1}^{-}\right]_{+}=-2 i\left(D_{2} K_{2}+K_{2} D_{2}\right)+2 K_{2}\left(\alpha_{2}+2 \Sigma\right), \quad\left[S_{2,2}^{-}, S_{2,1}^{+}\right]_{+}=\left(\left[S_{2,2}^{+}, S_{2,1}^{-}\right]_{+}\right)^{\dagger},}
\end{gathered}
$$

where $\alpha_{2}$ is given by Eq. (3.2) with $n=2$. Comparing with classical relations, we see that the anticommutation relations are obtained from the corresponding Poisson brackets (2.20), 
(2.21), (2.22) by symmetrization of the product of the noncommuting so $(1,2)$ generators and by the quantum shift $\alpha \rightarrow \alpha-\frac{1}{2}$.

In general case of supersymmetry of the order $n$, one can easily find the following anticommutation relations:

$$
\begin{gathered}
{\left[S_{n, n}^{+}, S_{n, n}^{-}\right]_{+}=\left(2 K_{n}\right)^{n},} \\
{\left[S_{n, n}^{+}, S_{n, n-1}^{-}\right]_{+}=-i\left(D_{n}\left(2 K_{n}\right)^{n-1}+\left(2 K_{n}\right)^{n-1} D_{n}\right)+\left(2 K_{n}\right)^{n-1}\left(\alpha_{n}+n \Sigma\right) .}
\end{gathered}
$$

According to relation (3.16), the quantum analog of the classical Poisson bracket relations is obtained by the same quantization prescription as in the simplest nonlinear case $n=2$ : by symmetrizing the product of noncommuting operators and by changing $\alpha$ for $\alpha_{n}$. However, comparing the quantum relation

$$
\begin{aligned}
{\left[S_{n, n-1}^{+}, S_{n, n-1}^{-}\right]_{+}=} & \frac{1}{2}\left(2 H_{n}(2 K)^{n-1}+\left(2 K_{n}\right)^{n-1} 2 H_{n}\right)+(n-1)(n-2)\left(2 K_{n}\right)^{n-2} \\
& +4(n-1)(2 K)^{n-2} \alpha_{n} \Sigma,
\end{aligned}
$$

with the corresponding classical relation (2.20) taken for $m=l=n-1$, one concludes that due to the presence of the second term, the same quantization prescription (a simple symmetrization of the noncommuting factors accompanied by the shift of the parameter $\alpha$ ) does not work in general case (note, that the additional term in (3.17) vanishes for the simplest nonlinear case $n=2$ ). Similar complications appear also in writing the explicit structure of other anticommutators of the odd generators for the general case of the nonlinear superconformal symmetry of the order $n$. On the other hand, the anticommutator of not depending explicitly on time odd integrals is, like Eq. (3.15), the direct quantum analog of the corresponding classical Poisson bracket relations:

$$
\left[S_{n, 0}^{+}, S_{n, 0}^{-}\right]_{+}=\left(2 H_{n}\right)^{n} .
$$

To conclude the discussion of the structure of the quantum nonlinear superconformal algebra, we prove the relation (3.18). The two terms on the left hand side of Eq. (3.18) may be written in the form

$$
\text { l.h.s. }=(-1)^{n} \mathcal{P}_{\alpha, n} \mathcal{P}_{-\alpha+n-1, n} \Pi_{+}+(-1)^{n} \mathcal{P}_{-\alpha+n-1, n} \mathcal{P}_{\alpha, n} \Pi_{-},
$$

where in correspondence with the notation used above $\Pi_{ \pm}=\frac{1}{2}\left(1 \pm \sigma_{3}\right), \Pi_{ \pm}^{2}=\Pi_{ \pm}, \Pi_{+} \Pi_{-}=0$, $\Pi_{+}+\Pi_{-}=1$. Subsequently using the relation

$$
\mathcal{D}_{\gamma} \mathcal{D}_{-\gamma}=\mathcal{D}_{-(\gamma-1)} \mathcal{D}_{\gamma-1},
$$

Eq. (3.19) may be represented as

$$
\text { l.h.s. }=\left(-\mathcal{D}_{-(\alpha-n)} \mathcal{D}_{\alpha-n}\right)^{n} \Pi_{+}+\left(-\mathcal{D}_{-\alpha} \mathcal{D}_{\alpha}\right)^{n} \Pi_{-} .
$$

The Hamiltonian (3.1) has an equivalent representation

$$
H_{n}=\frac{1}{2}\left(-\frac{d^{2}}{d x^{2}}+\frac{j_{n}\left(j_{n}+1\right)}{x^{2}}\right), \quad j_{n}=\alpha-n \Pi_{+},
$$

from which we get the equalities $2 H_{n} \Pi_{+}=-\mathcal{D}_{-(\alpha-n)} \mathcal{D}_{\alpha-n} \Pi_{+}$and $2 H_{n} \Pi_{-}=-\mathcal{D}_{-\alpha} \mathcal{D}_{\alpha} \Pi_{-}$, and so, reduce Eq. (3.19) to the right hand side of Eq. (3.18). 


\section{Two related nonlinear superconformal symmetries}

Relation (3.10) means that for the special value of the parameter, $\alpha=n-1$, the equality $H_{n}=H_{n-1}$ takes place. This means that the corresponding system may be characterized simultaneously by the nonlinear superconformal symmetries of the orders $n$ and $n-1$. Let us investigate the nature of such systems and the associated supersymmetries. To do this, we first investigate the question what is the most general case of the quantum systems (3.1) possessing such a property. If the system can be characterized by the supersymmetries of different orders $n$ and $n^{\prime}$, its Hamiltonian $H_{n}$ has to admit the alternative representation in the form of the Hamiltonian $H_{n^{\prime}}$ corresponding in general case to a different value of the parameter $\alpha$. So, let us require that

$$
H_{n}(\alpha)=H_{n^{\prime}}\left(\alpha^{\prime}\right)
$$

Having in mind that Eq. (4.1) can be satisfied in two ways, $H_{n}^{ \pm}(\alpha)=H_{n^{\prime}}^{ \pm}\left(\alpha^{\prime}\right)$, or $H_{n}^{ \pm}(\alpha)=$ $H_{n^{\prime}}^{\mp}\left(\alpha^{\prime}\right)$, where the index $+(-)$ corresponds to the $+1(-1)$ eigensubspace of $\sigma_{3}$, we find the following possible cases:

$$
\begin{gathered}
\alpha=\alpha^{\prime}=\frac{1}{2}\left(n+n^{\prime}-1\right), \\
\alpha=-\left(\alpha^{\prime}+1\right)=\frac{1}{2}\left(n-n^{\prime}-1\right) .
\end{gathered}
$$

The Hamiltonian in these two cases is given by

$$
H=\frac{1}{2}\left(-\frac{d^{2}}{d x^{2}}+\frac{\left(n \pm n^{\prime}\right)^{2}-1}{4 x^{2}} \mp \frac{n n^{\prime}}{x^{2}} \Pi_{+}\right),
$$

where the upper and lower signs correspond to the cases (4.2) and (4.3). Two other cases are given by the relations

$$
\begin{gathered}
\alpha-n=-\left(\alpha^{\prime}+1\right)=\frac{1}{2}\left(n+n^{\prime}+1\right), \\
\alpha-n=\alpha^{\prime}=\frac{1}{2}\left(n-n^{\prime}+1\right), \\
H=\frac{1}{2}\left(-\frac{d^{2}}{d x^{2}}+\frac{\left(3 n+2 \pm n^{\prime}\right)^{2}-1}{4 x^{2}}-\frac{n\left(2 n+2 \pm n^{\prime}\right)}{x^{2}} \Pi_{+}\right) .
\end{gathered}
$$

Comparing (4.4) with (4.7), we see that the second Hamiltonian can be obtained from the first one via the formal change $n^{\prime} \rightarrow n^{\prime} \mp 2(n+1)$. Note that the case (3.10) is a particular case of (4.2) with $n^{\prime}=n-1$, and that in all the cases (4.2), (4.3), (4.5), (4.6) the model parameter $\alpha$ takes integer or half-integer values.

Let us consider some particular cases. When $n^{\prime}=0$, the last term in Eq. (4.4) turns into zero, and the Hamiltonian takes a pure bosonic form

$$
H=\frac{1}{2}\left(-\frac{d^{2}}{d x^{2}}+\frac{n^{2}-1}{4 x^{2}}\right) .
$$

This corresponds to a trivial supersymmetry with odd integrals of motion $\psi^{+}$and $\psi^{-}$. This is in correspondence with Eqs. (3.1), (3.2) according to which the Hamiltonian takes a pure 
bosonic form $\left(b_{n}=0\right)$ only for $\alpha=\frac{1}{2}(n-1)$, i.e. when $\alpha$ takes an integer or a half-integer value. On the other hand, this system can be characterized by the nonlinear superconformal supersymmetry of the order $n$. Since $\psi^{ \pm}$are the odd integrals of motion, the corresponding even operators appearing as factors in odd integrals (3.5), (3.6) are the integrals of motion. Let us clarify their nature. For this, subsequently using the factorization relations

$$
-\frac{d^{2}}{d x^{2}}+\frac{n^{2}-1}{4 x^{2}}=-\mathcal{D}_{\frac{1}{2}(n+1)} \mathcal{D}_{-\frac{1}{2}(n+1)}=-\mathcal{D}_{-\frac{1}{2}(n-1)} \mathcal{D}_{\frac{1}{2}(n-1)}
$$

we find that in the case of $n=2 p$ the odd integral $S_{2,0}^{+}$is equal to $(-2 H)^{p} \psi^{+}$. The same happens with other odd integrals of motion: they are reduced to the products of the trivial odd integrals $\psi^{ \pm}$and of the even integrals $H, D$ and $K$. Therefore, in the case $n=2 p$ the order $n$ supersymmetry of the system (4.8) is trivial.

The pure bosonic system (4.8) with odd $n=2 p+1$ is more interesting. In this case the integral of motion $\mathcal{P}_{p, 2 p+1}$ presenting as a factor in odd integral $S_{2 p+1,0}^{+}$is a a local differential operator of order $2 p+1$ which effectively is the $p+\frac{1}{2}$ degree of the Hamiltonian. For instance, for $n=5$ this follows from the following chain of relations due to a subsequent application of Eq. (4.9):

$$
\begin{gathered}
\left(\mathcal{P}_{2,5}\right)^{2}=\mathcal{D}_{-2} \mathcal{D}_{-1} \mathcal{D}_{0} \mathcal{D}_{1}\left(\mathcal{D}_{2} \mathcal{D}_{-2}\right) \mathcal{D}_{-1} \mathcal{D}_{0} \mathcal{D}_{1} \mathcal{D}_{2}=\mathcal{D}_{-2} \mathcal{D}_{-1} \mathcal{D}_{0} \mathcal{D}_{1}\left(\mathcal{D}_{-1} \mathcal{D}_{1}\right) \mathcal{D}_{-1} \mathcal{D}_{0} \mathcal{D}_{1} \mathcal{D}_{2}= \\
\mathcal{D}_{-2} \mathcal{D}_{-1} \mathcal{D}_{0} \mathcal{D}_{0}^{2} \mathcal{D}_{0}^{2} \mathcal{D}_{0} \mathcal{D}_{1} \mathcal{D}_{2}=\mathcal{D}_{-2} \mathcal{D}_{-1}\left(\mathcal{D}_{1} \mathcal{D}_{-1}\right)^{3} \mathcal{D}_{1} \mathcal{D}_{2}= \\
\mathcal{D}_{-2}\left(\mathcal{D}_{2} \mathcal{D}_{-2}\right)^{4} \mathcal{D}_{2}=\left(\mathcal{D}_{3} \mathcal{D}_{-3}\right)^{5}=(-2 H)^{5}
\end{gathered}
$$

Analogously, other odd operators, $S_{n, l}^{ \pm}, l=1, \ldots, n$, supply us with the even polynomial differential operators being effectively the half-integer degrees of the operators $H^{n-m} K^{m}$ (in this symbolic writing we neglect the quantum ordering problem).

One can observe that in general cases of (4.2), (4.3), (4.5) and (4.6) with $n^{\prime} \neq 0, n>n^{\prime}$, the following relation takes place:

$$
S_{n, 0}^{+}=\mathcal{A}_{n, n^{\prime}} S_{n^{\prime}, 0}^{+} .
$$

When $n-n^{\prime}=2 p, p=1,2, \ldots$, this relation reduces to $S_{n, 0}^{+}=(-2 H)^{p} S_{n^{\prime}, 0}^{+}$, and higher nonlinear supersymmetry of order $n$ is a trivial derivative of the lower supersymmetry. The simplest example of such a case is given by Eqs. (4.2) and (4.4) with $n=3, n^{\prime}=1, \alpha=\alpha^{\prime}=$ $\frac{3}{2}$, for which, with taking into account Eq. (4.9), we have the relation $2 H \psi^{+}=-\mathcal{D}_{-\frac{1}{2}} \mathcal{D}_{\frac{1}{2}} \psi^{+}$, and, as a consequence, $S_{3,0}^{+}=-2 H S_{1,0}^{+}$. On the other hand, the cases (4.2), (4.3), (4.5) and (4.6) with $n^{\prime} \neq 0, n-n^{\prime}=2 p+1$ give the systems with the both nonlinear supersymmetries of the orders $n^{\prime}$ and $n=n^{\prime}+2 p+1$ to be nontrivial. As an illustration, one can consider the system with $n^{\prime}=n-1=\alpha$. Such a system is given by the Hamiltonian

$$
H=\frac{1}{2}\left(-\frac{d^{2}}{d x^{2}}+\frac{n(n-1)}{x^{2}} \Pi_{-}\right)
$$

and is characterized by the two superconformal symmetries of the orders $n$ and $n-1$. Note that the upper component $H^{+}$of the Hamiltonian (4.12) corresponds to the free particle system. The anticommutators of the supercharges corresponding to the $n$ and $n-1$ supersymmetries produce even nontrivial integrals of motion. For the simplest case of $n=2$, 
there is the relation $S_{2,0}^{+}=\mathcal{D}_{0} S_{1,0}^{+}$. Since $2 H \psi^{+}=-\mathcal{D}_{0}^{2} \psi^{+}$, we see that here the role of the additional factor in $S_{2,0}^{+}$is reduced effectively to multiplication of the supercharge $S_{1,0}^{+}$by the square root from the Hamiltonian. The anticommutator of the supercharges $S_{2,0}^{+}$and $S_{1,0}^{-}$ produces the even integral of motion,

$$
\left[S_{2,0}^{+}, S_{1,0}^{-}\right]_{+}=-\left(\mathcal{D}_{0}^{3}-\frac{3}{x^{2}} \mathcal{D}_{-1} \Pi_{-}\right) \equiv \mathcal{R},
$$

and the direct calculation shows that the third order local differential operator $\mathcal{R}$ has a nature of the $3 / 2$ degree of the Hamiltonian: $\mathcal{R}^{2}=(-2 H)^{3}$. Analogously, other anticommutators of the $S_{2, l}^{ \pm}, l=0,1,2$, with $S_{1, l^{\prime}}^{ \pm}, l^{\prime}=0,1$, produce even integrals being effectively half-integer degrees of the third order polynomials of the $s o(1,2) \times u(1)$ generators.

The considered example of the system (4.12) with $n^{\prime}=1$ and $n=2$ belongs to the general class of the systems given by the Hamiltonian of the form

$$
H=\frac{1}{2}\left(-\frac{d^{2}}{d x^{2}}+\frac{p}{x^{2}}\left(p-\sigma_{3}\right)\right), \quad p \in \mathbb{N} .
$$

The Hamiltonian (4.13) is a particular case of the system (4.2), (4.4) with $n^{\prime}=1$ and $n=2 p$, and is the direct quantum analog of the classical system (2.6) with $\alpha=p$. In addition to the usual superconformal symmetry of the order $n^{\prime}=1$, the quantum system (4.13) possesses the nonlinear superconformal symmetry of the order $n=2 p$, the odd generators of which produce, via the anticommutators with the fermionic $n^{\prime}=1$ superconformal symmetry generators, the additional nontrivial bosonic integrals of motion having a nature of the halfinteger degrees of the odd order polynomials of the $s o(1,2) \times u(1)$ generators.

\section{Discussion and outlook}

We have showed that a simple change of the boson-fermion coupling constant $2 \alpha \rightarrow 2 \alpha n$, $n \in \mathbb{N}$, in the classical superconformal mechanics model (2.6) gives rise to a radical change of the symmetry. Instead of the linear Lie superalgebraic $\operatorname{osp}(2 \mid 2)$ structure associated with the model (2.6), the modified system (2.10) is characterized by the nonlinear superconformal symmetry of the order $n$. The latter is generated by the four even integrals forming the $s o(1,2) \times u(1)$ Lie subalgebra, and by the two complex conjugate sets of the $(n+1)$ odd integrals forming the spin- $\frac{n}{2}$ representations of the $s o(1,2)$ subalgebra and anticommuting (in the Poisson bracket sense) for the polynomials of the order $n$ of even generators.

We have demonstrated that there exists the anomaly free quantization of the modified system preserving all the set of the integrals and the nonlinear superalgebraic structure. On the other hand, due to the quantum corrections a new, in comparison with the classical case, phenomenon arises at the quantum level: when the model parameter $\alpha$ takes an integer value, the quantum system is characterized simultaneously by the two nonlinear superconformal symmetries of the orders relatively shifted in odd number (see, however, ref. [29] where the classical analog of such a situation was discussed and refs. [27, 36], where a similar phenomenon was discussed at the quantum level for some systems with $N=1$ nonlinear supersymmetry). The anticommutators of the generators from these two different fermionic sets produce new nontrivial bosonic local integrals of motion being effectively the half-integer 
degrees of the odd order polynomials of the $s o(1,2) \times u(1)$ generators. In particular case, when the Hamiltonian has a purely bosonic form (4.8), one of the corresponding supersymmetries of the order $n^{\prime}=0$ is trivial (the corresponding odd integrals are reduced to the fermionic operators $\psi^{ \pm}$), and fermionic generators of another supersymmetry of the order $n=2 p+1$ are reduced to the compositions of the conserved fermionic operators $\psi^{ \pm}$and of additional nontrivial bosonic integrals. (When $n=2 p$, the even factors in the corresponding fermionic generators of the order $n=2 p$ supersymmetry are reduced to the products of the $s o(1,2)$ generators, i.e. the additional nonlinear superconformal symmetry is trivialized). Another interesting particular case of the systems with the two supersymmetries is given by the Hamiltonian (4.13). This quantum family, being, on the one hand, a quantum analog of the original system (2.6) with $\alpha=p, p \in \mathbb{N}$, possesses a usual $n^{\prime}=1$ superconformal symmetry and, on the other hand, is characterized simultaneously by the nonlinear superconformal symmetry of the order $n=2 p$.

Recently it has been shown $[10,11,17,21]$ that the "relativistic" generalization of the superconformal mechanics model which governs the motion of a superparticle near the AdS horizon of an extreme Reissner-Nordström black hole [5] is canonically equivalent to the original model $[2,3]$. Therefore, for the appropriate values of the parameters of the "relativistic" superconformal mechanics system of ref. [5] (when the corresponding boson-fermion coupling constant takes an even integer value), at the quantum level in addition to the linear $\operatorname{ssp}(2 \mid 2)$ Lie superalgebraic symmetry it possesses a nontrivial nonlinear superconformal symmetry of the even order.

To conclude, let us list some problems which deserve a further attention.

The superconformal mechanics model describes the relative motion of the two-particle Calogero system. Therefore, it would be interesting to investigate whether the $N$-particle

supersymmetric Calogero system [38, 8, 12, 39] admits a generalization for the case of the nonlinear supeconformal symmetry. Another interesting problem is a geometric realization of the described nonlinear superconformal symmetry on the appropriate superspace. This, in particular, could help in clarifying the question of the superfield formulation for the model (2.10). The obtained results may also be developed in application to other supersymmetric systems possessing superconformal symmetries, e.g., to the fermion-monopole system [40, 41]. We hope to present the results of the analysis of these problems elsewhere.

\section{Acknowledgements}

We thank D. Sorokin for a useful comment. The work has been supported in part by FONDECYT-Chile (grant 1010073) (M.P.), and by CONICYT-Chile (C.L.).

Note added. In the subsequent paper [42], the origin of the quantum corrections to the integrals of motion of the model (2.10) is clarified, and the complete form of the quantum nonlinear superconformal algebra of the arbitrary order $n$ is fixed.

\section{References}

[1] V. de Alfaro, S. Fubini and G. Furlan, Nuovo Cim. A 34 (1976) 569. 
[2] V. P. Akulov and A. I. Pashnev, Teor. Mat. Fiz. 56 (1983) 344.

[3] S. Fubini and E. Rabinovici, Nucl. Phys. B 245 (1984) 17.

[4] E. A. Ivanov, S. O. Krivonos and V. M. Leviant, J. Phys. A 22 (1989) 345; J. Phys. A 22 (1989) 4201.

[5] P. Claus, M. Derix, R. Kallosh, J. Kumar, P. K. Townsend and A. Van Proeyen, Phys. Rev. Lett. 81 (1998) 4553 [hep-th/9804177].

[6] J. A. de Azcarraga, J. M. Izquierdo, J. C. Perez Bueno and P. K. Townsend, Phys. Rev. D 59 (1999) 084015 [hep-th/9810230].

[7] R. Kallosh, "Black holes and quantum mechanics," hep-th/9902007.

[8] G. W. Gibbons and P. K. Townsend, Phys. Lett. B 454 (1999) 187 [hep-th/9812034].

[9] J. Michelson and A. Strominger, Commun. Math. Phys. 213 (2000) 1 [hep-th/9907191].

[10] J. Kumar, JHEP bf 9904 (1999) 006 [hep-th/9901139].

[11] S. Cacciatori, D. Klemm and D. Zanon, Class. Quant. Grav. 17 (2000) 1731 [hepth/9910065].

[12] N. Wyllard, J. Math. Phys. 41 (2000) 2826 [hep-th/9910160].

[13] R. Britto-Pacumio, J. Michelson, A. Strominger and A. Volovich, "Lectures on superconformal quantum mechanics and multi- black hole moduli spaces," hep-th/9911066.

[14] E. E. Donets, A. Pashnev, V. O. Rivelles, D. P. Sorokin and M. Tsulaia, Phys. Lett. B 484 (2000) 337 [hep-th/0004019].

[15] G. Papadopoulos, Class. Quant. Grav. 17 (2000) 3715 [hep-th/0002007].

[16] M. S. Plyushchay, Nucl. Phys. B 589 (2000) 413 [hep-th/0004032].

[17] M. Cadoni, P. Carta and S. Mignemi, Phys. Rev. D 62 (2000) 086002 [hep-th/0004107].

[18] A. Marcus, JHEP 0102 (2001) 043 [hep-th/0101017].

[19] E. Deotto, G. Furlan and E. Gozzi, Phys. Lett. B 481 (2000) 315 [hep-th/0204108].

[20] I. Bandos, E. Ivanov, J. Lukierski and D. Sorokin, JHEP 0206 (2002) 040 [hepth/0205104].

[21] E. Ivanov, S. Krivonos and J. Niederle, "Conformal and superconformal mechanics revisited," hep-th/0210196;

S. Bellucci, A. Galajinsky, E. Ivanov and S. Krivonos, Phys. Lett. B 555 (2003) 99 [hep-th/0212204];

E. Ivanov, S. Krivonos and O. Lechtenfeld, JHEP 0303 (2003) 014 [hep-th/0212303]. 
[22] C. Leiva and M. S. Plyushchay, Ann. Phys. (NY) 307 (2003) 372 [hep-th/0301244].

[23] O. Aharony, S. S. Gubser, J. M. Maldacena, H. Ooguri and Y. Oz, Phys. Rept. 323 (2000) 183 [hep-th/9905111].

[24] A. A. Andrianov, M. V. Ioffe and V. P. Spiridonov, Phys. Lett. A 174 (1993) 273 [hepth/9303005];

A. A. Andrianov, M. V. Ioffe and D. N. Nishnianidze, Phys. Lett. A 201 (1995) 103, [hep-th/9404120];

A. A. Andrianov, F. Cannata, J. P. Dedonder and M. V. Ioffe, Int. J. Mod. Phys. A 10 (1995) 2683 [hep-th/9404061].

[25] B. F. Samsonov, Mod. Phys. Lett. A 11 (1996) 1563 [quant-ph/9611012].

[26] D. J. Fernandez C, Int. J. Mod. Phys. A 12 (1997) 171 [quant-ph/9609009].

[27] A. A. Andrianov, F. Cannata, M. Ioffe and D. Nishnianidze, Phys. Lett. A 266 (2000) 341 [quant-ph/9902057].

[28] M. Plyushchay, Int. J. Mod. Phys. A 15 (2000) 3679 [hep-th/9903130].

[29] S. M. Klishevich and M. S. Plyushchay, Nucl. Phys. B 606 (2001) 583 [hep-th/0012023].

[30] H. Aoyama, M. Sato and T. Tanaka, Phys. Lett. B 503 (2001) 423-429, [quantph/0012065]; Nucl. Phys. B 619 (2001) 105-127 [quant-ph/0106037];

H. Aoyama, N. Nakayama, M. Sato and T. Tanaka, Phys. Lett. B 519 (2001) 260-268 [hep-th/0107048].

[31] S. M. Klishevich and M. S. Plyushchay, Nucl. Phys. B 628 (2002) 217 [hep-th/0112158].

[32] S. M. Klishevich and M. S. Plyushchay, Nucl. Phys. B 616 (2001) 403 [hep-th/0105135]; Nucl. Phys. B 640 (2002) 481 [hep-th/0202077];

[33] S. M. Klishevich and M. S. Plyushchay, J. Phys. A 36 (2003) 11299 [hep-th/0212117].

[34] P. Dorey, C. Dunning and R. Tateo, J. Phys. A 34 (2001) 5679-5704 [hep-th/0103051].

[35] F. Cannata, M. V. Ioffe and D. N. Nishnianidze, J. Phys. A 35 (2002) 1389-1404 [hepth/hep-th/0201080].

[36] A. A. Andrianov and A. V. Sokolov, Nucl. Phys. B 660 (2003) 25 [hep-th/0301062].

[37] E. Witten, Nucl. Phys. B 188 (1981) 513.

[38] D. Z. Freedman and P. F. Mende, Nucl. Phys. B 344 (1990) 317.

[39] S. Bellucci, A. Galajinsky and S. Krivonos, Phys. Rev. D 68 (2003) 064010 [hepth/0304087].

[40] E. D'Hoker and L. Vinet, Phys. Lett. B 137 (1984) 72. 
[41] M. S. Plyushchay, Phys. Lett. B 485 (2000) 187 [hep-th/0005122].

[42] A. Anabalon and M. S. Plyushchay, Phys. Lett. B 572 (2003) 202 [arXiv:hepth/0306210]. 